\title{
Review of "Concept Maps"
}

\author{
Andy Bytheway ${ }^{1}$ \\ 1 University of the Western Cape
}

The idea of concept mapping has been around for decades but has never found the currency that it deserves. The brief explanation provided suggests strongly the idea from business systems analysis of "Entity relationship modelling" (ERM) which is an approach that I have generalised to achieve simple, conceptual representations of a domain of knowledge. However, ERMs are not hierarchical, and have rules about the establishment and naming of relationships. For example, they have *direction*, which is not indicated in the examples provided in the linked article; further, the idea of cardinality of the relationships between concepts (one-to-one, one-to-many and many-to-many) has to be acknowledged and represented if we are going to avoid representational chaos in a particular domain. A many-to-many relationship has to be resolved, because it masks embedded associative concepts that must be identified and added to the model. Further, I would usually choose to name all concepts with a noun (or a noun phrase) and all relationships with a verb (or a verb phrase).

In my view, this definition is extremely important and needs development, agreement and stabilisation. 